\title{
Short Summaries
}

\author{
Derek Richards \\ Centre for Evidence Based Dentistry, Oxford UK
}

These short summaries are of Cochrane and other systematic reviews

Evidence-Based Dentistry (2007) 8, 27-28. doi:10.1038/sj.ebd.6400476

\section{Antiretroviral postexposure prophylaxis}

Young TN, Arens FJ, Kennedy GE, Laurie JW, Rutherford GW. Antiretroviral post-exposure prophylaxis (PEP) for occupational HIV exposure. Cochrane Database of Systematic Reviews 2007; issue 1

The dental team, like other healthcare workers, is at risk from known or potentially HIV_contaminated products. The estimated risk for HIV infection depends on the site and nature of the exposure. The Centers for Disease Control in Atlanta (Georgia, USA) estimate the risk of HIV infection via a percutaneous needlestick from a known HIV-infected source to be $0.3 \%$.

The risk of seroconversion is influenced by: an increased volume of blood contaminant because of a deep injury or procedures involving a needle placed directly in vein or artery; an increased HIV viral load in the source patient; and failure to administer a form of postexposure prophylaxis (PEP). This Cochrane review sought to evaluate the effects of antiretroviral PEP after occupational exposure to HIV.

No randomised controlled trials were identified. Only one case-control study was included. HIV transmission was significantly associated with deep injury [odds ratio (OR), 15; 95\% confidence intervals $(\mathrm{Cl}), 6.0-41.0]$; visible blood on the device (OR, 6.2; $95 \%$ $\mathrm{Cl}, 2.2-21.0)$; procedures involving a needle placed in the source patient's blood vessel (OR, 4.3; $95 \% \mathrm{Cl}, 1.7-12.0)$; and terminal illness in the source patient (OR, 5.6; $95 \% \mathrm{Cl}, 2.0-16.0)$. After controlling for these risk factors, no differences were detected in the rates at which cases and controls were offered PEP with zidovudine. Cases had significantly lower odds of having taken zidovudine after exposure than controls (OR, $0.19 ; 95 \% \mathrm{Cl}, 0.06-0.52)$. No studies were found that evaluated the effect of two or more antiretroviral drugs for occupational PEP.

It is highly unlikely that definitive placebo-controlled trials will ever be conducted because of ethical and practical issues; on the basis of results from a single case-control study the authors recommend that a 4-week regimen of PEP should be initiated as soon as possible after exposure, depending on the risk of seroconversion.

Despite the fact that there is no direct evidence to support multidrug antiretroviral regimens following occupational exposure to HIV, the authors recommend that because of the success of combination therapies in treating HIV-infected individuals, a combination of antiretroviral drugs should be used for PEP. The authors also recommend counselling healthcare workers about expected adverse events and the strategies for managing these, as well as advising that PEP is not $100 \%$ effective in preventing HIV seroconversion.

\section{Acupuncture for neck disorders}

Trinh KV, Graham N, Gross AR, et al.

Acupuncture for neck disorders. Cochrane Database of Systematic Reviews 2006; issue 3

Acupuncture is sometimes used as an alternative to more traditional treatments for musculoskeletal pain. The review included 10 trails (661 participants) that examined the effects of acupuncture on neck pain for individuals who had chronic neck pain (lasting for at least 3 months). One study also included individuals with neck pain that lasted for at least 6 weeks but they considered it to be chronic. Acupuncture was compared with being on the waiting list for acupuncture, sham acupuncture, other sham treatments (sham laser, sham transcutaneous electric nerve stimulation; TENS or other treatments (mobilisation, massage, traction). Acupuncture treatments appear to be safe and only minor, transient and benign adverse effects were reported in the trials.

The trials were of moderate methodological quality, but the number of participants in each trial was relatively low. There was a range of individuals studied, acupuncture techniques used and outcomes measured, so the results of the trials could not be combined to obtain an overall picture of the effectiveness of acupuncture. Therefore, only limited conclusions could be drawn. Individuals who had chronic neck pain and who received acupuncture reported, on average, better pain relief immediately after treatment and in the short-term than those who received sham treatments. Individuals who had chronic neck pain with symptoms radiating to the arms who received acupuncture reported, on average, better pain relief in the short-term than those who were on a waiting list.

\section{Medicinal and Injection therapies for mechanical neck disorders}

Peloso P, Gross A, HainesT, et al.

Medicinal and injection therapies for mechanical neck disorders. Cochrane Database of Systematic Reviews 2005; issue 2

This Cochrane review examined the effect of medicines and injections on pain, function/disability, patient satisfaction and range of motion in participants who had mechanical neck disorders (MND).

Thirty-two trials that examined the effects of oral nonsteroidal anti-inflammatory drugs (NSAID), psychotropic agents, injections of steroids, and anaesthetic agents were included. 
Intramuscular injection of lidocaine for chronic MND and intravenous injection of methylprednisolone for acute whiplash were found to be effective treatments. There was limited evidence for effectiveness of epidural injection of methylprednisolone and lidocaine for chronic MND with radicular findings. Oral psychotropic agents had mixed results. There was moderate evidence that botulinum toxin intramuscular injections for chronic MND were no better than saline. Other medications, including NSAID, had contradictory or limited evidence of effectiveness.

\section{Treatments for basal cell carcinoma}

Bath-Hextall FJ, Perkins W, Bong J, Williams HC.

Interventions for basal cell carcinoma of the skin. Cochrane Database of Systematic Reviews 2007; issue 1

This Cochrane review looked at the effectiveness of treatments for basal cell carcinoma (BCC). BCC is the most common skin cancer, $85 \%$ of cases appearing in the head and neck region. Although not life-threatening, BCC can destroy the skin and neighbouring tissues, causing significant cosmetic disfigurement, especially on the face. Treatments include surgical removal, radiotherapy, cryotherapy (freezing), phototherapy (light therapy) and creams.

A detailed search strategy was followed and studies of adults with one or more histologically proven, primary BCC were included. The primary outcome measure was recurrence at 3-5 years, measured clinically.

Twenty-seven studies were identified. Only one randomised controlled trial of surgery versus radiotherapy had primary outcome data at 4 years, showing significantly more persistent tumours and recurrence in the radiotherapy group than in the surgery group [relative risk (RR), $0.09 ; 95 \%$ confidence interval $(\mathrm{Cl}), 0.01-0.69]$. One study found no significant difference for recurrence at 30 months when Moh's micrographic surgery was compared with surgery for high-risk facial BCC (RR, 0.64; 95\% Cl, 0.16-2.64). One study of methylaminolevulinate (MAL) photodynamic therapy (PDT) versus cryotherapy found no significant difference between recurrence in the MAL PDT group than following cryotherapy at 1 year (RR, $0.50 ; 95 \% \mathrm{Cl}, 0.22-1.12$ ). Cryotherapy showed no significant difference in recurrence at 1 year compared with surgery in one small study. When radiotherapy was compared with cryotherapy there were significantly fewer recurrences at 1 year in the radiotherapy group than in the cryotherapy group. Short-term studies suggest a success rate of $87-88 \%$ for imiquimod cream in the treatment of superficial BCC using a once-daily regimen for 6 weeks and a $76 \%$ treatment response when treating nodular BCC for 12 weeks, when measured histologically.

The authors found very little good-quality research on treatments for $B C C$, with most trials only evaluating BCC in low-risk locations. Surgery and radiotherapy appear to be the most effective treatments: surgery shows the lowest failure rates. Although cosmetic outcomes appear to be good with PDT, long-term follow-up data are needed. Other treatments might have some use but few have been compared with surgery. An ongoing study testing imiquimod cream versus surgery should clarify whether imiquimod is a useful option.

\section{Hangover cures: do they work?}

\section{Pittler MH, Verster JC, Ernst E.}

Interventions for preventing or treating alcohol hangover: systematic review of randomised controlled trials. Br Med J 2005; 331;1515-1518

Hangovers have extensive economic and health costs and the number of suggested cures on offer is extensive. A search on Google (search term: hangover cures; accessed 5 November 2006) elicited over 1 million hits, giving an indication of the number of suggestions available. The aim of this review was clinical evidence on the effectiveness of any medical intervention for preventing or treating alcohol hangover.

Systematic searches were conducted using Medline, Embase, Amed (Allied and Complementary Medicine Database), Cochrane Central, the National Research Register (UK), and ClincalTrials.gov (US). Searches were made by hand of conference proceedings and bibliographies, and experts and manufacturers of commercial preparations were contacted. There were no language restrictions.

Randomised controlled trials (RCT) of any medical intervention for preventing or treating alcohol-induced hangovers were included. Placebo-controlled trials or those trials that controlled against a comparator intervention were included.

Study selection, data extraction and validation were done independently by two reviewers with the Jadad score being used to evaluate methodological quality.

Only 15 potentially relevant trials were identified. The paucity of RCT is in marked contrast to the plethora of hangover cures marketed on the internet. Eight double-blind RCT were reviewed testing a variety of agents, namely propranolol, tropisetron, tolfenamic acid, fructose or glucose, and dietary supplements made from Borago officinalis (borage), Cynara scolymus (artichoke), Opuntia ficus-indica (prickly pear), and a yeast-based preparation. Significant intergroup differences for overall symptom scores and individual symptoms were reported only for tolfenamic acid, linolenic acid from B. officinalis, and the yeastbased preparation.

The authors conclude that there is no compelling evidence to suggest that any conventional or complementary intervention is effective for preventing or treating alcohol hangover. Although normally this would lead for calls for more research, the authors highlight possible ethical concerns because positive trials might lead to considerable media interest and industry marketing, which ultimately might lead to an increase in alcohol consumption. There is, in fact, little evidence to show that alleviation of hangover symptoms results in increased alcohol consumption. ${ }^{1}$

The authors also suggest that further studies should investigate the biological changes that occur during alcohol hangover, for until the pathology of the alcohol-induced hangover is understood in more detail, an effective intervention is likely to remain elusive. In the mean time, the most effective way to avoid the symptoms of alcohol induced hangover is thus to practise abstinence or moderation.

1. Wiese JG, Shlipak MG, Browner WS. The alcohol hangover. Ann Intern Med 2000; 132:897-902. 